\title{
An RNA Interference-Based Screen of Transcription Factor Genes Identifies Pathways Necessary for Sensory Regeneration in the Avian Inner Ear
}

\author{
David M. Alvarado, ${ }^{1}$ R. David Hawkins, ${ }^{1}$ Stavros Bashiardes, ${ }^{1}$ Rose A. Veile, ${ }^{1}$ Yuan-Chieh Ku, ${ }^{1}$ Kara E. Powder, ${ }^{1}$ \\ Meghan K. Spriggs, ${ }^{2}$ Judith D. Speck, ${ }^{2}$ Mark E. Warchol, ${ }^{2}$ and Michael Lovett ${ }^{1}$ \\ ${ }^{1}$ Division of Human Genetics, Department of Genetics, and ${ }^{2}$ Department of Otolaryngology and Central Institute for the Deaf, Washington University \\ School of Medicine, St Louis, Missouri 63110
}

\begin{abstract}
Sensory hair cells of the inner ear are the mechanoelectric transducers of sound and head motion. In mammals, damage to sensory hair cells leads to hearing or balance deficits. Nonmammalian vertebrates such as birds can regenerate hair cells after injury. In a previous study, we characterized transcription factor gene expression during chicken hair cell regeneration. In those studies, a laser microbeam or ototoxic antibiotics were used to damage the sensory epithelia (SE). The current study focused on 27 genes that were upregulated in regenerating SEs compared to untreated SEs in the previous study. Those genes were knocked down by siRNA to determine their requirement for supporting cell proliferation and to measure resulting changes in the larger network of gene expression. We identified 11 genes necessary for proliferation and also identified novel interactive relationships between many of them. Defined components of the $W N T, P A X$, and $A P 1$ pathways were shown to be required for supporting cell proliferation. These pathways intersect on WNT4, which is also necessary for proliferation. Among the required genes, the CCAAT enhancer binding protein, CEBPG, acts downstream of Jun Kinase and JUND in the AP1 pathway. The WNT coreceptor LRP5 acts downstream of CEBPG, as does the transcription factor BTAF1. Both of these genes are also necessary for supporting cell proliferation. This is the first large-scale screen of its type and suggests an important intersection between the AP1 pathway, the $P A X$ pathway, and WNT signaling in the regulation of supporting cell proliferation during inner ear hair cell regeneration.
\end{abstract}

\section{Introduction}

The inner ear is comprised of the vestibular and auditory sensory organs. Within the vestibular system, the utricle senses linear acceleration and head orientation to maintain balance. The cochlea is the auditory organ and detects sound. The cochlea and the vestibular organs use a small population of sensory hair cell (HCs) as mechanoelectric transducers. Loss of inner ear hair cells is the most frequent cause of human deafness and balance disorders (Frolenkov et al., 2004). Sensory hair cells are surrounded by nonsensory supporting cells (SCs). Both cell types originate from the same lineage and together comprise the sensory epithelia (SEs). The mammalian inner ear lacks the ability to regenerate sensory hair cells

Received Oct. 18, 2010; revised Nov. 30, 2010; accepted Feb. 2, 2011.

This work was supported by a grant from the National Organization for Hearing Research Foundation and National Institutes of Health Grant R01DC005632 to M.L. and R01DC006283 to M.E.W. Support for microscopy and imaging was provided by P30DC04665. We thank Dr. Anne M. Bowcock for critical reading of this manuscript.

R. D. Hawkins's present address: Ludwig Institute for Cancer Research, University of California School of Medicine, 9500 Gilman Drive, La Jolla, CA 92093.

S. Bashiardes's present address: Department of Molecular Virology, Cyprus Institute of Neurology and Genetics, International Airport Road, 1683 Nicosia, Cyprus.

Correspondence should be addressed to Michael Lovett, Division of Human Genetics, Department of Genetics, Washington University School of Medicine, 4566 Scott Avenue, St. Louis, M0 63110. E-mail: lovett@ genetics.wustl.edu.

DOI:10.1523/JNEUROSCI.5456-10.2011

Copyright $\odot 2011$ the authors $\quad 0270-6474 / 11 / 314535-09 \$ 15.00 / 0$ when damaged, but birds and other lower vertebrates are capable of regenerating sensory hair cells throughout their life (Corwin and Cotanche, 1988; Jørgensen and Mathiesen, 1988; Ryals and Rubel, 1988; Weisleder and Rubel, 1993).

The specific signaling pathways required for triggering sensory hair cell regeneration have yet to be identified. In this study, we characterized transcription factor (TF) genes that are differentially expressed during avian sensory HCs regeneration. These were identified in a gene expression study in which we measured changes in gene expression for $>1500$ TF genes across two different time courses of in vitro $\mathrm{HC}$ regeneration (Messina et al., 2004; Hawkins et al., 2007). One time course measured TF expression changes following laser microbeam injury. The second time course measured TF changes as the SEs regenerated after antibiotic ablation of the HCs (Warchol, 1999, 2001). These time courses were conducted on multiple pure SEs dissected from the cochlea and utricles of chickens. From this regeneration dataset, seven "known" pathways were identifiable: TGF- $\beta, P A X$, NOTCH, WNT, NFKB, Insulin/IGF, and AP1. A large number of TF changes were also identified for genes that have not yet been placed into established pathways. Together, this list of "known" and "unknown" TF genes was the starting point for the current study that focused upon testing their role in early regenerative proliferation. A major limitation in the restoration of sensory function in the human inner ear is the inability of the SCs to proliferate and differentiate into new sensory HCs in response to 
damage. In the current study, we focus on the genetic pathways required for the earliest stages of the regenerative process, specifically sensory epithelium proliferation. We used siRNA knockdown and treatment with small molecule inhibitors to test 27 genes for their effects upon early stages of avian regenerative proliferation. We identified 11 components that are necessary for the early steps in the regenerative process and identified individual components and new pathway intersections within the AP-1, $P A X$, and $W N T$ pathways that appear to be important effectors of $\mathrm{SC}$ proliferation.

\section{Materials and Methods}

Tissue dissections. Ten to twenty-one day posthatch White Leghorn chicks were killed via $\mathrm{CO}_{2}$ asphyxiation and decapitated. Utricles were explanted, and after incubation for $1 \mathrm{~h}$ in $500 \mu \mathrm{g} / \mathrm{ml}$ thermolysin, the SEs were removed from the stromal tissue. A detailed description of culture methods has appeared previously (Warchol, 2002).

Laser ablation. Fragments of sensory epithelia were cultured for 7-10 d on laminin-coated wells (Mat-Tek) that contained $50 \mu$ lof Medium$199 / 10 \%$ FBS. Semiconfluent cultures were then lesioned via laser microsurgery (Hawkins et al., 2007). Laser-lesioned protocol was initially performed for JNK, JunD, PAX2, and CEBPG and replicated with the dissociated utricle sensory epithelia protocol. All subsequent siRNA treatments were performed with the dissociated utricle sensory epithelia protocol.

Dissociated utricle sensory epithelia. Utricle sensory epithelia were physically dissociated into small fragments, pooled, and plated at a final concentration of 0.5 utricles per well in 96-well cultures to ensure that total cell density is uniform between compared samples. Cultures were grown for $3 \mathrm{~d}$ and transfected before confluency with siRNAs (50 ng/ well) or inhibitor in $0.1 \%$ DMSO ( $15 \mu \mathrm{M}$ SP600125 JNK inhibitor) using previously described methods (Elbashir et al., 2002).

siRNA generation. Double-stranded RNA (dsRNA) was generated by first PCR amplifying a portion of the gene of interest from chicken SE cDNA (supplemental Table S9, available at www.jneurosci.org as supplemental material). PCR products were amplified using gene-specific primers containing the $5^{\prime}$ T7 promoter sequence CTCTAATACGACTCACTATAGGG, under the following conditions: $100 \mathrm{ng}$ of cDNA, 0.2 $\mu \mathrm{M}$ (final concentration) each primer, $10 \times$ Advantage Taq Buffer (BD Biosciences), and $5 \mathrm{U}$ of Advantage Taq (BD Biosciences) in a final volume of $50 \mu \mathrm{l}$, incubated at $95^{\circ} \mathrm{C}$ for $2 \mathrm{~min}$, followed by 30 cycles of $95^{\circ} \mathrm{C}$ for $30 \mathrm{~s}, 55^{\circ} \mathrm{C}$ for $30 \mathrm{~s}$, and $68^{\circ} \mathrm{C}$ for $2 \mathrm{~min}$. PCR products were verified by DNA sequencing. Promoter-containing PCR products were used as template DNA in in vitro transcription (IVT) reactions (Ambion). IVT reactions, including postreaction DNase treatment and precipitation, were performed according to the manufacturer's protocol for $12 \mathrm{~h}$. Equal amounts (typically $3 \mu \mathrm{g}$ each) of sense and antisense RNA strands were mixed and heated at $75^{\circ} \mathrm{C}$ for $10 \mathrm{~min}$ and brought to room temperature on the bench for $2 \mathrm{~h}$. dsRNAs were treated with RNase ONE (50 U, Promega) for $45 \mathrm{~min}$ at $37^{\circ} \mathrm{C}$. dsRNA was cleaned using RNA Purification Columns 1 (Gene Therapy Systems). siRNAs were generated using the Dicer enzyme (Gene Therapy Systems) following the manufacturer's protocol. Dicer-generated siRNA (d-siRNA) was checked on a $3 \%$ agarose gel for $\sim 23 \mathrm{bp}$ size. d-siRNA was cleaned up using RNA Purification Columns 2 (Gene Therapy Systems). Regions used to generate these target sequences were computationally compared to the chicken genome using custom PERL scripts and NCBI Blast. Only regions containing no more than $14 \mathrm{bp}$ of nonspecific sequence overlap in 21 bp sliding windows were used for these siRNA treatments. PCR products were amplified using gene-specific primers containing the $5^{\prime}$ T7 promoter sequence. These were used as template DNA in IVT reactions (Ambion). Fifty nanograms of d-siRNA were transfected in each well of dissociated SE cultures or laser microbeam-ablated SE cultures using standard Lipofectamine 2000 (Invitrogen) protocols. siRNA treatments that inhibited sensory epithelium proliferation were independently replicated with chemically synthesized Dicer-substrate RNAs (DsiRNAs) obtained from Integrated DNA Technologies predesigned DsiRNA Library when available or custom designed (supplemental Table S10, available at www. jneurosci.org as supplemental material) and transfected using standard Lipofectamine 2000 (Invitrogen) protocols. All significantly altered transcripts after siRNA treatments were computationally scanned for possible off-target sequence homologies with the siRNA products.

Confirmation of siRNA knockdown. Knockdown of target siRNAs were determined by expression profiling of each siRNA knockdown or by endpoint quantitative RT-PCR (JunD, PAX5, BCL11A, TRIP15, and $M Y T 1 L$ ) when microarray expression values were not statistically significant due to dye effects on microarray probe performance. For microarray expression confirmation, knockdowns are defined as $>1.3$-fold decrease in expression with $p<0.05$ across all replicates.

Proliferation index. Cells were assayed $48 \mathrm{~h}$ after transfection using previously published protocols (Warchol and Corwin, 1996). Quantification of cell proliferation was measured by calculating a proliferation index (defined as the number of BrdU+ cells/total cells). Cells from $10,000 \mu \mathrm{m}^{2} \times 3$ regions of a 96-well plate were combined to determine the proliferation index per well with a minimum of four wells per biological samples. Proliferation assays were replicated with independently dissociated sensory epithelia and siRNA transfections, with a minimum of two biological samples per treatment. Mean proliferation indexes were determined using ImageJ 1.36b software (http://rsb.info.nih.gov/ij/), and error bars were generated by calculating the SD of proliferation indexes across all wells and biological samples. Differences between control values in experimental groups most likely reflect minor changes in initial plating density (0.5 utricles/well) and BrdU labeling efficiency between different experimental groups. To control for these variations, each experimental group was compared to a control sample that was plated, transfected, labeled, and counted in parallel.

Exogenous WNT4 treatment. Chicken utricle sensory epithelia were physically dissociated and plated as previously described. Mouse WNT4 protein (R\&D Systems) was initially added at 15, 50, and $100 \mathrm{ng} /$ well in $100 \mu \mathrm{l}$ of media. Mouse WNT5A protein (R\&D Systems) was assayed at $100 \mathrm{ng} /$ well in $100 \mu \mathrm{l}$ of media. Cells were assayed $48 \mathrm{~h}$ after treatment using previously published protocols (Warchol and Corwin, 1996).

Microarray hybridizations and analysis. RNA isolation and cDNA synthesis was performed as previously described (Hawkins et al., 2003). Microarray comparisons were Lowess normalized, and genes with intensity below background as determined by control spots were removed. All comparative microarray hybridizations consisted of a minimum of two biological samples and four technical replicates for each biological sample, including dye switch experiments. A one-sample $t$ test was used to determine statistically significant changes in gene expression $(p<0.05)$ across all replicates for each treatment. All microarray data for the current study has been deposited with NCBI GEO with accession number GSE16842.

\section{Results}

A high-throughput, quantitative measure of SE proliferation The design of our previous TF gene expression study (Hawkins et al., 2007) is summarized in Figure $1 A$. TFs identified as being upregulated in that discovery set were moved into the current study, which is diagrammed in Figure $1 B$. This consisted of testing individual components for their effects on cell proliferation and on gene expression. We initially used laser lesioning to damage SEs and measured the effects of siRNA or small molecule inhibitors to stop regenerative proliferation (left side of Fig. $1 B$; Fig. 2). This slow and qualitative assay was replaced by a higherthroughput and quantitative assay, which used cultures of dissociated utricular SCs (right side of Fig. 1B). RNA interference (RNAi) and inhibitor treatments that blocked repair of a laserlesioned SE also showed similar patterns of proliferative inhibition in our 96-well assays. This suggests that our higher-throughput assay system correctly identified a subset of genes that are necessary for regenerative proliferation in the intact SE. All quantitative proliferation results and expression profiling presented here were performed with the dissociated utricle SE protocol. 
A

B

Transfect siRNA

SE on coverslip
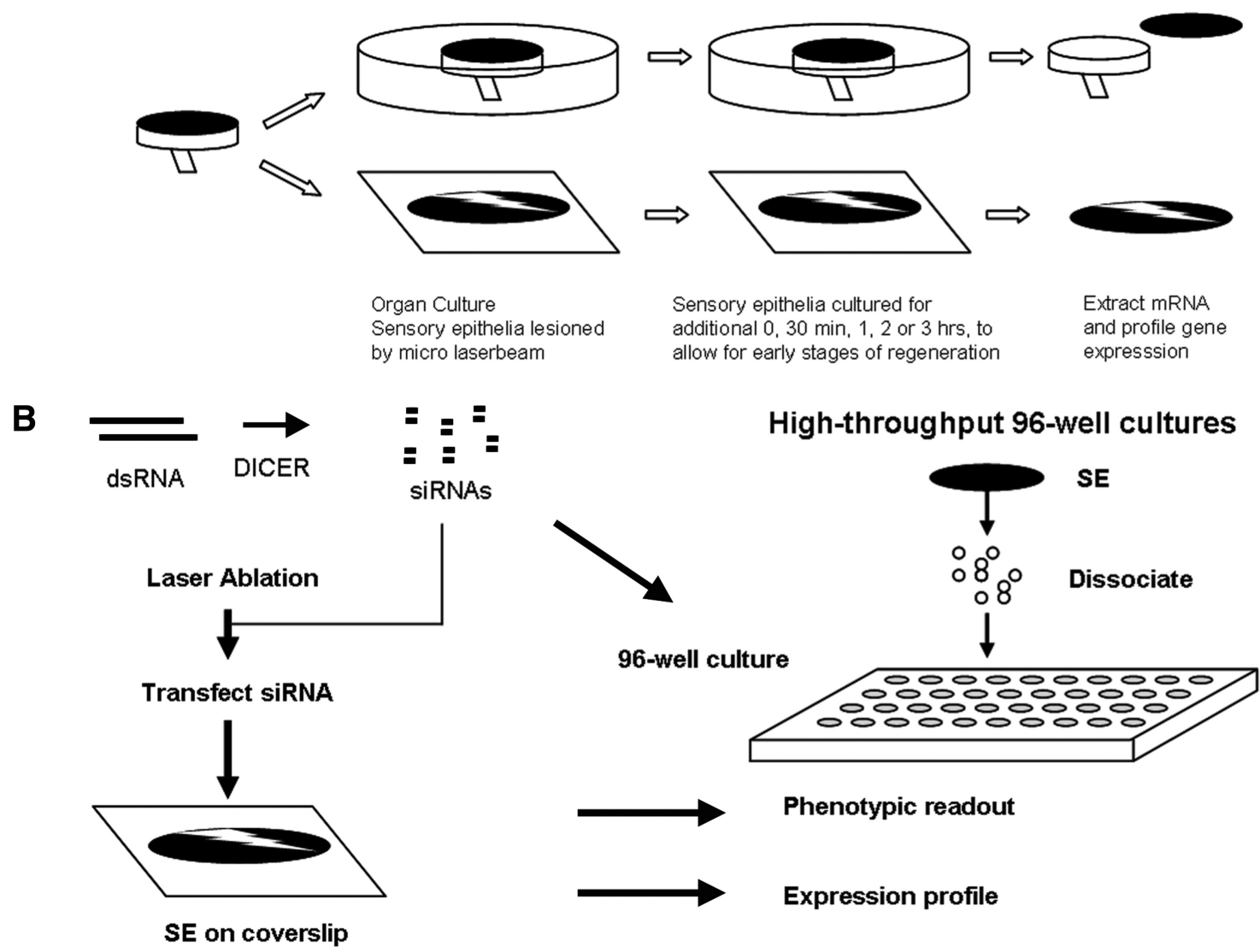

Organ Culture

Hair cells lesioned by addition

of $1 \mathrm{mM}$ neomycin for $24 \mathrm{hr}$.
Organs cultured for additional

0,1 , or 2 days, to allow for

early stages of regeneration
Remove pure sensory epithelia. Extract mRNA and profile gene expresssion

Figure 1. Experimental design. Flow diagram of experimental design scheme for time course profiling in the utricle and cochlea SE and RNAi profiling. $A$, Time course of laser and neomycin recovery. $\boldsymbol{B}$, TFs revealed in the time course of recovery were targeted by siRNA to assess a proliferation phenotype and expression profiled to evaluate knockdown of the target gene and potential epistatic relationships between TFs.
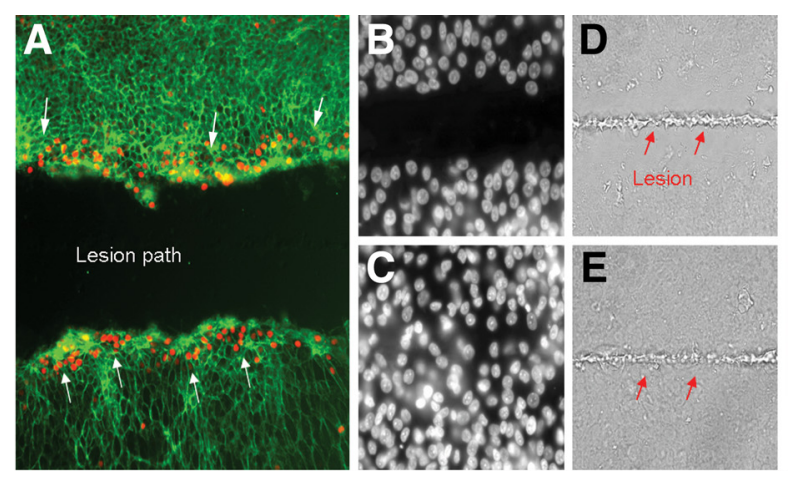

Figure 2. JNK signaling during SE regeneration. JNK signaling is evident at the leading edge of the lesion path in the SEs and necessary for proliferative regeneration. SE cultured on a glass coverslip was lesioned by microbeam laser ablation. $A$, Phosphorylated C-JUN was detected by a phosphorylation-specific antibody to the protein (red dots; white arrows). B, C, Following laser ablation, the cultured SE was treated with JNK inhibitor (SP600125, $15 \mu \mathrm{M})(\boldsymbol{B})$ or $0.1 \%$ DMSO (control) ( $($ ) and allowed to recover for $24 \mathrm{~h}$; nuclei are shown by DAPI staining. $\boldsymbol{D}, \boldsymbol{E}$, The laser lesion path is visible by etching of the coverslip through the phase contrast ( $\boldsymbol{D}$ and $\boldsymbol{E}$, red arrows). Only the JNK inhibitor exhibited a failure to close the wound.

Genes necessary for regenerative proliferation

A complete list of siRNA and small molecule inhibitor treatments and their effects on SE proliferation is given in Table 1 (discussed below). All RNAi knockdowns were confirmed by microarray expression profiling or endpoint quantitative PCR. Cellular proliferation was assessed by BrdU labeling and is expressed as a
JNK Inhibitor (SP600125 $15 \mu \mathrm{M}$ )

Control (0.1\% DMSO) "proliferation index," defined as the number of BrdU-labeled nuclei/total DAPIlabeled nuclei per microscopic field.

Our previous work suggested that HC regeneration is regulated (in part) by the activating protein 1 (AP1) complex that includes the JUN family of TFs (Hawkins et al., 2007). JUN proteins can be induced by a large number of signaling molecules, as well as by physical or chemical stress (Shaulian and Karin, 2002). Ten known components of the AP1 pathway were differentially expressed during SE regeneration (Hawkins et al., 2007). To determine whether activation of JUN occurs after SE injury, we conducted immunohistochemical staining on laser-lesioned utricular SEs, using an antibody specific to the phosphorylated form of $c$-JUN (Fig. 2A). Phosphorylated $c$-JUN was detected at both leading edges of the laser lesion site. To test whether the initial activation of the JUN family of TFs is necessary for SE regeneration, we treated the laser-lesioned SE with a small molecule inhibitor (SP600125; $15 \mu \mathrm{M}$ ) of the JUN activator, c-JUN-N-terminal kinase (JNK) (Bennett et al., 2001; Heo et al., 2004; Assi et al., 2006). This led to a failure in regen- 
Table 1. Effects of siRNA/inhibitor treatments on sensory epithelia proliferation

\begin{tabular}{llll}
\hline & Inhibit & Average fold & \\
siRNA/inhibitor treatment & proliferation & knockdown & Regeneration pathway/category \\
\hline CEBPG & Yes & -3.92 & AP-1 pathway \\
JNK inhibitor & Yes & & \\
JUND & Yes & $*$ & \\
BTAF1 & Yes & -1.57 & AP-1 siRNA commonalities \\
LRP5 & Yes & -5.71 & \\
RARA & Yes & -1.41 & \\
PAX2 & Yes & -1.61 & Pax pathway \\
PAX3 & No & -1.61 & \\
PAX5 & Yes & $*$ & \\
PAX7 & No & -1.72 & \\
MYT1L & No & $*$ & AP-1/Pax siRNA commonalities \\
WNT4 & Yes & -2.27 & \\
CUTL1 & Yes & -1.86 & Cell cycle \\
P27KIP & No & -2.93 & \\
ID1 & No & -1.48 & \\
CBX3 & No & -4.15 & Polycomb complex \\
CBX4 & No & -1.09 & \\
EZH2 & No & -1.87 & \\
IGF inhibitor & No & & Pathway inhibitors \\
MAPK inhibitor & Yes & & \\
SHH inhibitor & No & & \\
HRY & No & -1.30 & Notch signaling \\
BCL11A & No & -1.35 & Common to all tissues/damage \\
TRIP15 & No & -1.12 & \\
CTNNB1 & No & -2.39 & Common to cochlea and utricle \\
TIME & No & -1.16 & Early regeneration \\
PPARGC1 & No & -1.42 & Neomycin specific \\
\hline Por & & & \\
\hline & & & \\
& & &
\end{tabular}

Proliferation phenotypes were quantified for each siRNA knockdown. Inhibition was determined as a significantly lower proliferation index than a GFP siRNA control $(p<0.05)$. Knockdowns of siRNA targets were confirmed by microarray analysis or $\left({ }^{*}\right)$ endpoint semiquantitative $P C R$.

erative wound closure (Fig. 2 B). Treatment for $24 \mathrm{~h}$ with $15 \mu \mathrm{M}$ SP600125 reduced the proliferation levels of cultured SCs by $32 \%$ (relative to untreated controls; $p<0.001$ ), while treatment for $48 \mathrm{~h}$ reduced proliferation by $44 \%(p<0.001)$. In contrast, treatment with $10 \mu \mathrm{M}$ SB203580, a small molecule inhibitor of p38 (a MAP kinase not implicated in our previous studies), had no effect on SC proliferation. These results demonstrate that functional JNK signaling is required for repair in the utricular SE.

Members of the JUN family of TFs are thought to be constitutively expressed (Brivanlou and Darnell, 2002) with their activity regulated by phosphorylation via JNK. However, our data suggest some degree of transcriptional regulation, since we observed increased expression of JUN family members (and in particular JUND) during regeneration (Hawkins et al., 2007). To determine whether reducing JUND levels inhibited SC proliferation, we used RNAi targeted to chicken JUND. These resulted in reduced SC proliferation $48 \mathrm{~h}$ after siRNA treatment (Fig. $3 A$ ), confirming that a functional AP1 pathway is necessary for SC proliferation.

Seven components of WNT signaling are differentially expressed during SE regeneration, including $\beta$-catenin, a critical component of canonical WNT signaling (Hinck et al., 1994). We selected $\beta$-catenin for knockdown by siRNA (see more WNT components below). We also selected genes that did not necessarily fall within known pathways, but were upregulated during one or more time points of SE regeneration. One example is the CCAAT element binding protein $(C E B P G)$, which was upregulated at specific time points in utricular regeneration (Hawkins et al., 2007). We also identified BCL11A (a zinc finger gene associated with hematopoietic malignancies) (Medina and Singh, 2005; Singh et al., 2005) and TRIP15 (a component of the COP9 signa-
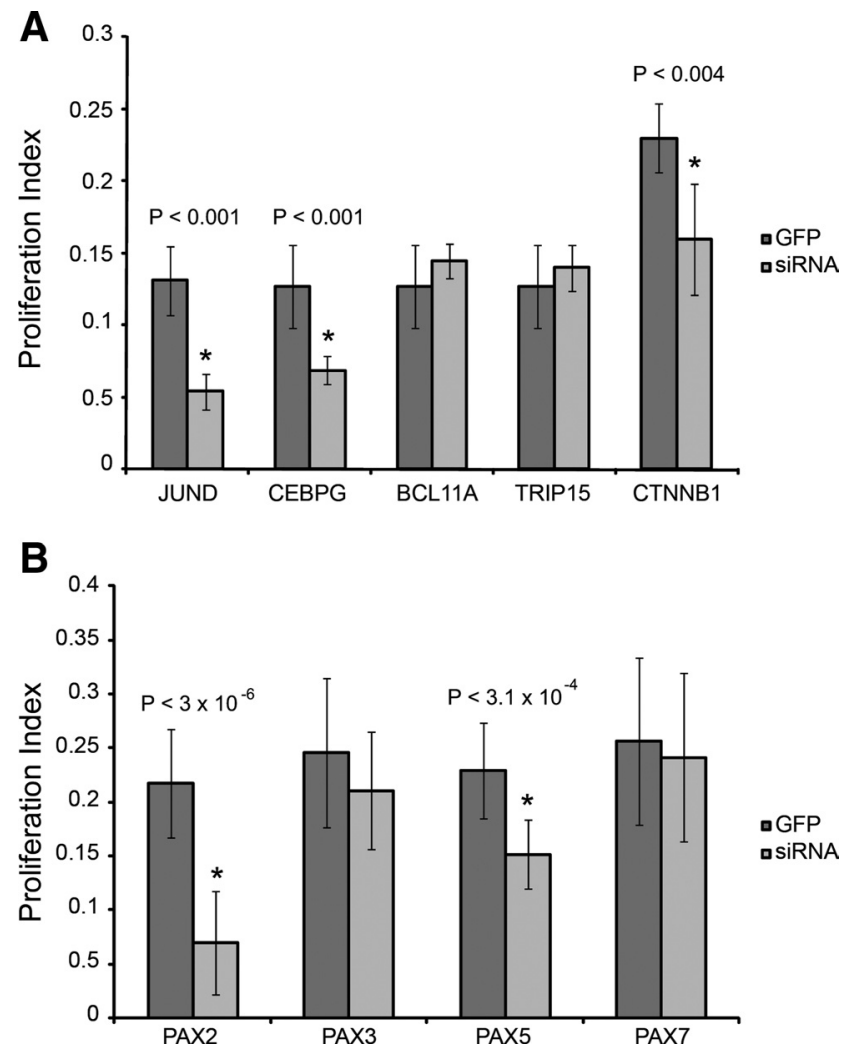

Figure 3. Effects of siRNA treatments on SC proliferation. Proliferation phenotypes were quantified for each siRNA knockdown compared to a GFP control by calculating a proliferation index. BrdU-labeled proliferating cells were compared to the total number of DAPI-stained cells to calculate a percentage proliferation for genes differentially expressed during $S E$ regeneration $(\boldsymbol{A})$ and PAX genes that were upregulated during SE regeneration $(\boldsymbol{B})$.

losome that regulates $\mathrm{G}_{1}-\mathrm{S}$ transition) (Yang et al., 2002) as being differentially expressed across all four treatment/tissue combinations (Hawkins et al., 2007). Knockdowns of CEBPG and $\beta$-catenin significantly reduced SC proliferation, similar to JUND siRNA treatments (Fig. 3A). However, siRNA knockdowns of BCL11A and TRIP15 failed to significantly affect SC proliferation.

The cyclin-dependent kinase $(C D K)$ inhibitor $p 27^{K i p l}$ is required for the transition in SCs from quiescence to the proliferative state (Chen and Segil, 1999; Löwenheim et al., 1999). The gene expression level of this CDK inhibitor within our regenerative time courses decreases $1 \mathrm{~h}$ after laser lesioning (Hawkins et al., 2007). Likewise, CUTL1 (a CCAAT displacement protein), a known $p 27^{K i p 1}$ repressor (Ledford et al., 2002), is differentially expressed across the time course. To determine whether $p 27^{\text {Kip } 1}$ and CUTL1 regulation are important regulators of SC proliferation, we used siRNAs targeted to each and measured the effects on cell proliferation. We reasoned that inhibition of CUTL1 would lead to a release of $p 27^{K i p 1}$ repression and a consequent decrease in proliferation. In agreement with this model, CUTL1 siRNA treatments inhibited SC proliferation and gene expression of $p 27^{\text {Kipl }}$ increased in these treatments (1.68-fold change, $p<$ $0.0176)$. However, siRNA knockdowns of $p 27^{\text {Kip } 1}$ had no apparent effect on proliferation (Table 1). This might be attributable to the already very high rate of ongoing cell division in these cultures (Kelley, 2006). However, as noted below, some other treatments can indeed result in SC hyperproliferation. Overall, these data are consistent with the known roles of CUTL1 and $p 27^{\text {Kip } 1}$ in the regulation of the transition from quiescence to the proliferative state. siRNA knockdowns of $I D-1$ upregulate $p 27^{\text {Kip } 1}$ and inhibit 

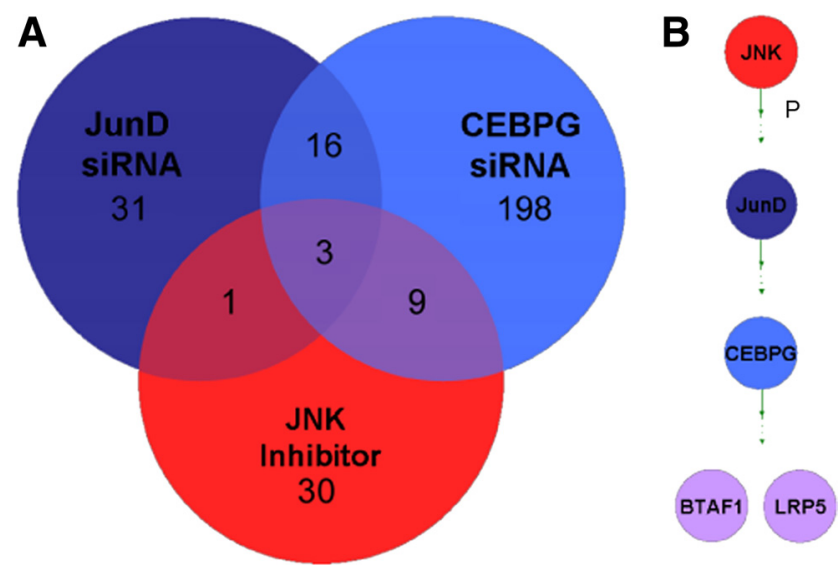

Figure 4. Analysis of overlapping expression profiles and novel epistatic relationships between genes that are necessary for $\mathrm{SC}$ proliferation. siRNA and inhibitor treatments were expression profiled to identify downstream effectors of $\mathrm{SC}$ proliferation. $\boldsymbol{A}$, Numbers indicate genes differentially expressed in three treatments that each individually inhibit $\mathrm{SC}$ proliferation. Three genes are commonly downregulated, one of which is CEBPG. B, Novel epistatic relationships can be inferred from TF expression profiling siRNA and inhibitor treatments. CEBPG can be placed downstream of JNK and JUND and the other commonly downregulated genes, BTAF1 and LRP5.

proliferation of mammalian tumors (Ling et al., 2002; Tam et al., 2008). However, our knockdowns of ID-1 had no effect on SC proliferation. Negative RNAi results of this type are open to the caveat that none of our knockdowns completely abrogated transcript levels. Thus, some small level of transcript (10-20\%) and protein product (not measured) may still be present and may be sufficient to maintain proliferation.

Our prior study revealed a cascade of $18 \mathrm{TF}$ genes induced by $P A X$ gene expression (Hawkins et al., 2007). Five $P A X$ genes (PAX2, PAX3, PAX5, PAX7, and PAX8) were upregulated during cochlear regeneration (Hawkins et al., 2007). To determine whether PAX genes are necessary for utricular SC proliferation, we used RNAi to knockdown these genes. A chick ortholog for $P A X 8$ could not be unequivocally identified, and it was therefore not targeted for knockdown. Approximately $10 \%$ of the chicken genome is missing from the published or web-accessible DNA sequence (International Chicken Genome Sequencing Consortium, 2004). This includes many genes that lack clear orthologs such as $P A X 8$, but are likely present in the chick genome. While most invertebrate genomes posses only a single $P A X 2 / 5 / 8$ gene, early in vertebrate evolution the closely related subclass of pairedbox family of TFs PAX2, PAX5, and PAX8 were produced by gene duplication (Noll, 1993; Mansouri et al., 1996; Czerny et al., 1997; Wada et al., 1998; Kozmik et al., 1999). From the four individual siRNA knockdowns, two ( $P A X 2$ and $P A X 5)$ inhibited SC proliferation, while knockdowns of $P A X 3$ and $P A X 7$ did not have significant effects on proliferation (Fig. $3 B$ ).

\section{Downstream effectors of sensory epithelium proliferation}

We conducted TF microarray expression profiling on all samples that were treated with siRNAs or inhibitors. This served the dual purpose of confirming the knockdown of the target gene and identifying additional genes that showed consistent expression changes in response to the treatment. We next identified overlapping expression changes between treatments. One example of such an intersection is shown in Figure $4 A$, which illustrates the TF expression changes for three treatments, all of which individually inhibit SC proliferation: JNK inhibitor, JUND RNAi, and CEBPG RNAi. While there are numerous expression changes that
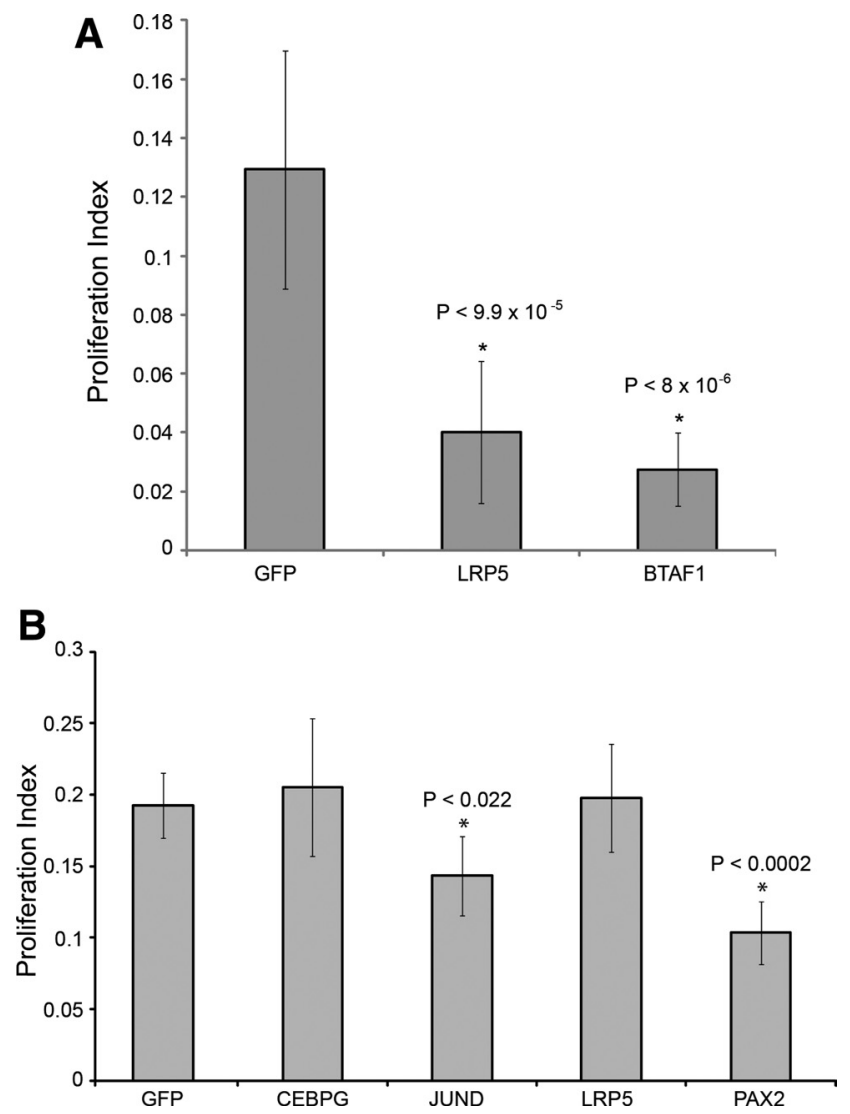

Figure 5. Analysis of siRNA treatments in chick SC and RPE proliferation. Percentage proliferation was quantified for siRNA treatments in chick SC for genes commonly downregulated in treatments that inhibit SC proliferation (downstream of the AP-1 pathway and (EBPG) $(\boldsymbol{A})$ and chick RPE for genes that inhibited chick SC proliferation $(B)$.

are unique to each treatment or shared between pairs of treatments, we identified three genes that are commonly downregulated in all three treatments (fold change $>1.3, p<0.05$ ) (supplemental Tables S1, S2, S3, available at www.jneurosci.org as supplemental material). One of these shared genes is CEBPG, suggesting that CEBPG acts downstream of JUND and JNK in this pathway. In addition, the low-density lipoprotein receptorrelated protein 5 gene ( $L R P 5$, a coreceptor of $W N T$ signaling) and the B-TFIID TF-associated RNA polymerase (BTAF1) were commonly downregulated in all three treatments, suggesting that these two genes probably act downstream of CEBPG in the JUN cascade (Fig. 4B). To determine whether these commonly downregulated genes are also required for SC proliferation, we conducted siRNA knockdowns of LRP5 and BTAF1. Both significantly inhibit SE proliferation (Fig. $5 A$ ).

\section{siRNA effects on proliferation that are specific to the inner ear}

To determine whether genes that regulate SC proliferation are also involved in the proliferation/repair of other types of epithelia, we performed RNAi knockdowns in cultures of chick retinal pigmented epithelium (RPE) (Fig. 5B) and measured proliferative indexes. Since JUND is the most broadly expressed TF of the AP1 pathway, it was not surprising to observe that siRNA knockdown of JUND also inhibited proliferation of chick RPE. Knockdowns of the widely expressed TF PAX2 also inhibited proliferation of RPE, suggesting that JUND and $P A X 2$ may serve common roles in both the ear and the eye. However, siRNA 
Table 2. Genes commonly differentially expressed in treatments that inhibit sensory epithelia proliferation

\begin{tabular}{|c|c|c|c|c|c|c|c|c|}
\hline \multirow[b]{2}{*}{ Gene } & \multicolumn{4}{|c|}{ Downstream of Ap-1 pathway } & \multicolumn{4}{|l|}{ Pax pathway } \\
\hline & CEBPG siRNA & $p$ value & LRP5 siRNA & $p$ value & PAX2 siRNA & $p$ value & PAX5 siRNA & $p$ value \\
\hline MYT1L & -4.27 & $7 \times 10^{-3}$ & -4.05 & $7.00 \times 10^{-3}$ & -1.51 & $2.00 \times 10^{-3}$ & -1.61 & $1.40 \times 10^{-2}$ \\
\hline WNT4 & 5.41 & $1.70 \times 10^{-2}$ & 4.16 & $3.90 \times 10^{-2}$ & 1.34 & $4.70 \times 10^{-2}$ & 1.37 & $7.00 \times 10^{-3}$ \\
\hline
\end{tabular}

Expression profiles for siRNA knockdowns that inhibited sensory epithelia proliferation were compared to identify specific commonalities downstream of the Ap-1 and PAX pathways. MYT1L and WNT4 were commonly differentially expressed (average fold change $>1.3, p<0.05$ ) in all four siRNA treatments that inhibit proliferation.

knockdowns of CEBPG and LRP5 had no effect on RPE proliferation, suggesting they may be uniquely required for SC proliferation in the inner ear.

\section{Pathways and pathway intersections}

To identify known pathways downstream of CEBPG and LRP5, we used MetaCore Analysis software (Ekins et al., 2006) to compare gene expression profiles derived from CEBPG and LRP5 siRNA knockdowns in dissociated utricular cultures (supplemental Tables S3, S4, S5, available at www.jneurosci.org as supplemental material). MetaCore Analysis is a web base tool that identifies components of known pathways enriched in our datasets. $p$ values are generated to determine the probability that genes are found by chance. One of the highest scoring pathways in both CEBPG and LRP5 siRNA knockdowns was the NOTCH signaling pathway $\left(p<8.89 \times 10^{-13}\right.$ and $p<7.48 \times 10^{-4}$, respectively). NOTCH signaling is known to regulate the differentiation of HCs and nonsensory SCs during inner ear development and HC regeneration (Adam et al., 1998; Lanford et al., 1999; Cotanche and Kaiser, 2010). We also identified enrichment of TGF- $\beta$ signaling $\left(p<6.18 \times 10^{-8}\right.$ and $\left.p<1.58 \times 10^{-7}\right)$ and WNT signaling $\left(p<1.01 \times 10^{-5}\right.$ and $\left.p<2.62 \times 10^{-5}\right)$. Components of both pathways are differentially expressed during SE regeneration (Hawkins et al., 2007). Three specific components of WNT signaling (WNT4, WNT9B, and WNT16) were commonly upregulated in both siRNA treatments $(>2$-fold change, $p<0.05$ ) (supplemental Tables S3, S5, available at www. jneurosci.org as supplemental material).

To determine whether potential pathway intersections can be discovered within our siRNA data, we compared gene expression profiles of four siRNA treatments that individually inhibited SC proliferation: CEBPG, LRP5, PAX2, and PAX5 siRNA. We identified two genes that are commonly upregulated or downregulated across all four siRNA treatments ( $>1.3$-fold change, $p<$ 0.05 ); these were the WNT gene family member (WNT4) and myelin TF 1-like (MYT1L) (Table 2, supplemental Tables S3, S5, S6, S7, available at www.jneurosci.org as supplemental material). To determine whether WNT4 and MYT1L are also necessary for SC proliferation, we used RNAi to knockdown each in cultured SEs. Knockdowns of MYT1L had no effect, but knockdown of WNT4 significantly reduced SC proliferation (Fig. 6). Upregulation of WNT4 occurred in six of eight treatments that reduced SC proliferation $(p<0.02)$ (supplemental Fig. S1, available at www. jneurosci.org as supplemental material), supporting a critical role for WNT4 during SC proliferation. Interestingly, five known components of NOTCH signaling (see below) were differentially expressed $(>1.5$-fold change and $p<0.05)$ in WNT4 siRNA knockdowns (supplemental Table S8, available at www. jneurosci.org as supplemental material). Progenitor cells of the sensory epithelia acquire either the HC or SC fate by lateral inhibition through the NOTCH signaling cascade. Progenitor HCs express elevated levels of the NOTCH ligand, DELTA, causing neighboring cells to increase expression of $\mathrm{NOTCH}$ (Adam et al., 1998; Morrison et al., 1999). Increased levels of NOTCH induce

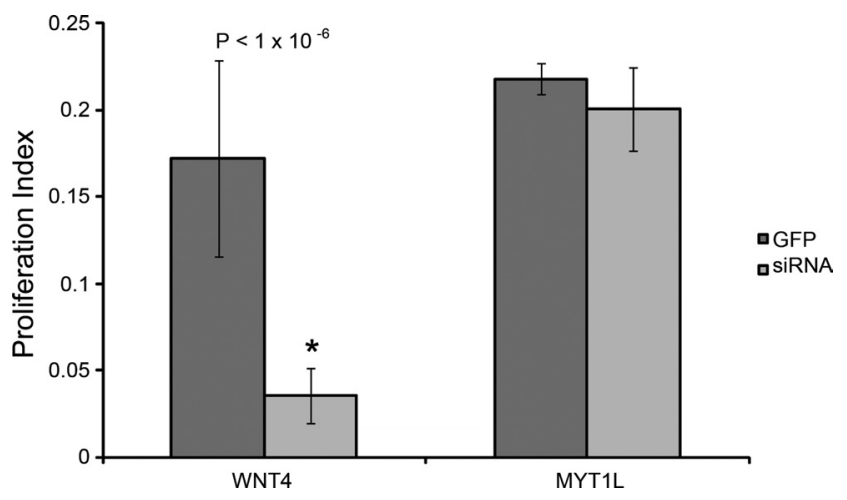

Figure 6. WNT4 and MYT1L siRNA phenotypes. WNT4 siRNA knockdowns inhibited SC proliferation compared to a GFP control, while MYT1L siRNA did not have a significant effect on proliferation.

Hairy and Enhancer of Split related genes, negatively regulating DELTA and inhibiting sensory HC fate (Zheng et al., 2000; Zine et al., 2001). In our WNT4 siRNA knockdowns, we identified downregulation of NOTCH1, NOTCH2, and HEY2 and upregulation of DELTA1 and DELTA3, suggesting that WNT4 may be involved in regulating NOTCH signaling. Recent reports have suggested a closely linked relationship between WNT and NOTCH ("WNTCH") signaling (Hayward et al., 2008). These reports suggest a model in which WNT signaling establishes a prepatterned group of cells capable of specific differentiation states, individual cell fates then being further refined by $\mathrm{NOTCH}$ signaling.

\section{Exogenous Wnt4 increases proliferation}

After establishing that WNT4 is necessary for SE proliferation, we next interrogated the effects of exogenous WNT4 protein on SE proliferation. To determine whether exogenous WNT4 would result in increased proliferation, we examined dissociated chick utricle SEs after incubation in WNT4-supplemented media. SEs cultured in WNT4 resulted in hyperproliferation compared to cultures grown in media supplemented with heat-inactivated WNT4 and unsupplemented media (Fig. 7A). Interestingly, cultures supplemented with the WNT ligand WNT5A also resulted in SE hyperproliferation (Fig. $7 B$ ), suggesting that the activation of WNT signaling is a critical step during SE proliferation and is not solely limited to the expression of WNT4.

\section{Discussion}

Our data suggest that, while the AP1 and $P A X$ pathways have unique downstream components, both pathways intersect at WNT4. Moreover, expression of WNT4 is also necessary for SC proliferation, pointing to a critical role for WNT signaling in the initiation of regeneration in the avian ear. WNT4 levels increase in response to siRNA treatments that inhibit SC proliferation. If increased WNT4 expression is inhibiting proliferation, then this would suggest that siRNA knockdowns of WNT4 might lead to normal or even hyperproliferation. However, we found that 


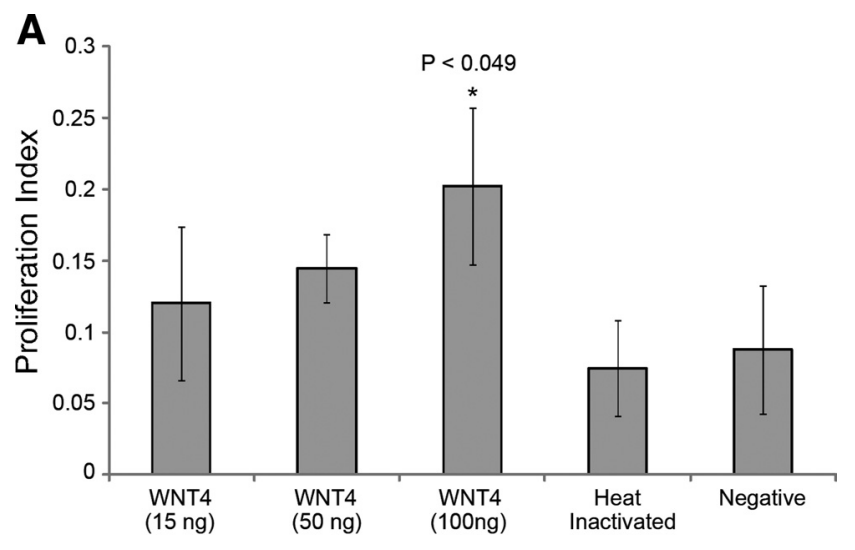

B

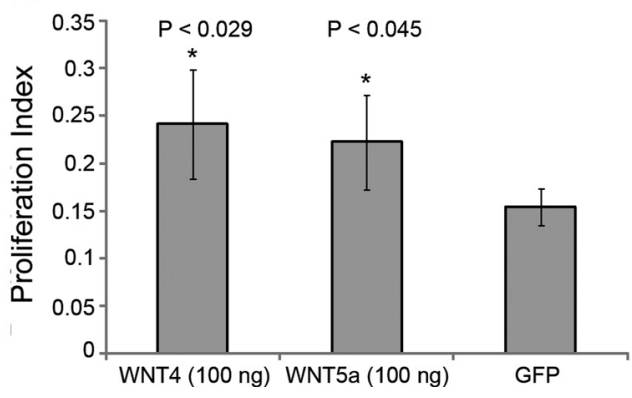

Figure 7. Exogenous WNT4 expression phenotypes. $\boldsymbol{A}$, Percentage proliferation was quantified for treatment with 15,50 , or $100 \mathrm{ng} /$ well of exogenous WNT4 protein. Cells treated with $100 \mathrm{ng} /$ well of exogenous WNT4 protein hyperproliferated compared to heat-inactivated WNT4 and cells cultured in unsupplemented media. $\boldsymbol{B}$, Treatment with exogenous WNT5a also caused hyperproliferation; however, CEBPG siRNA in combination with exogenous WNT4 treatment inhibited proliferation similarly to CEBPG siRNA treatment alone.

WNT4 siRNAs inhibited SC proliferation and exogenous WNT4 results in hyperproliferation. This suggests that while inhibition of the AP1 pathway and downstream components results in altered WNT signaling regulation, both CEBPG and WNT signaling are necessary for SE proliferation. Further investigation of this complex circuit will be necessary to resolve this apparent conundrum. It is interesting to note that WNT4 has been described in both the canonical and noncanonical WNT signaling pathways (Lyons et al., 2004; Miyakoshi et al., 2008) and appears to be capable of sequestering $\beta$-catenin at the cell surface (Chang et al., 2007). These apparently dual functions may be the basis for the complexity of the results when WNT4 levels are experimentally manipulated.

WNT4 is thought to act as an autoinducer of mesenchyme to epithelial transition during mouse kidney development (Stark et al., 1994). WNT4 null mice die within $24 \mathrm{~h}$ of birth due to kidney failure, precluding subsequent analysis of hearing or balance disorders (Stark et al., 1994). It is first detected in the developing chicken otocyst at E4, in the prospective nonsensory cochlear duct (Sienknecht and Fekete, 2009), and later at E5, forming a border between the sensory primordia and nonsensory lateral wall (Stevens et al., 2003; Sienknecht and Fekete, 2008), suggesting that WNT4 may play an important role in forming sensory/ nonsensory boundaries in the developing inner ear. During a regenerative time course after neomycin treatment, WNT4 expression increased at $72 \mathrm{~h}$ after the removal of neomycin (2.26fold change, $\left.p<5.77 \times 10^{-4}\right)$. This is at a time when the earliest known markers of $\mathrm{HC}$ regenerated via mitosis are first detected (Stone and Cotanche, 2007), suggesting that WNT4 may be in- volved in the early stages of SC to HC differentiation. PAX2 has been shown to regulate WNT4 expression during kidney development (Torban et al., 2006) and our microarray data suggest that $P A X 2$, along with $P A X 5, C E B P G$, and $L R P 5$, may function as important regulators of WNT4 in the inner ear. Thus, the AP1 and $P A X$ pathways both appear to affect $W N T$ signaling during $\mathrm{SE}$ regeneration.

CUTL1 is a known downstream transcriptional target of TGF- $\beta$ signaling (Michl et al., 2005; Hawkins et al., 2007) is upregulated during regeneration (Hawkins et al., 2007) and, as shown here, is necessary for SC proliferation. This gene was also downregulated in two treatments that inhibited SC proliferation (WNT4 and BTAF1 knockdowns; -1.90-fold and -1.38-fold changes, respectively) (supplemental Tables S8, S9, available at www.jneurosci.org as supplemental material), suggesting that regulation of CUTL1 may be important during SC proliferation. CUTL1 represses $p 27^{\text {Kip } 1}$, which is first detected in the sensory primordia of the developing mouse cochlea from E12 to E14, a time when proliferation is ending and HC differentiation is beginning (Chen and Segil, 1999). Expression of $p 27^{\text {Kip } 1}$ in the adult inner ear identifies cochlear SCs and participates in the inhibition of cell cycle entry in these cells (White et al., 2006). $p 27^{\text {Kip } 1}$ homozygous knock-out mice develop with an excess number of HCs and SCs (Chen and Segil, 1999; Löwenheim et al., 1999; Kanzaki et al., 2006). This suggests that $p 27^{\text {Kipl }}$ plays an important role in maintaining mitotically inactive sensory epithelium cells in mammals. $p 27^{\text {Kip }}$ is rapidly downregulated following injury to the avian utricle (Hawkins et al., 2007), possibly because of the increased expression of its repressor, CUTL1. Removal from the cell cycle plays an important role in maintaining functionally active SEs. This may be an important factor in the lack of regenerative capabilities in mammalian SEs.

JUN family TFs also play important roles in regulating cell cycle entry, proliferation, and differentiation. For example, $c$-JUN can remove p53-mediated inhibition of cell cycle entry (Shaulian et al., 2000) and JUND regulates mouse lymphocyte proliferation (Meixner et al., 2004). Members of the JUN family of TFs interact with FOS to activate Cyclin D1 and increase cell proliferation (Shaulian and Karin, 2002). Ten components of the AP1 complex, including FOS, were differentially expressed in one or more of our regenerative time points (Hawkins et al., 2007). Our data place $C E B P G$ downstream in the $A P 1$ pathway during sensory regeneration. This is further supported by the recent observation that $C E B P G$ interacts with FOS to activate the $I L-4$ gene in Jurkat cells (Davydov et al., 1995). CEBPG belongs to the highly conserved CCAAT/enhancer binding protein $(C / E B P)$ family of TFs. Members of this family act as master regulators of numerous processes, including differentiation, inflammatory response, and liver regeneration (Ramji and Foka, 2002). It is possible that CEBPG interacts with FOS or other members of the AP1 complex to regulate proliferation during avian utricular regeneration.

Our results indicate that $L R P 5$ also acts downstream in the $A P 1$ pathway during sensory regeneration. The $L R P 5$ protein can function as a coreceptor in WNT signaling (Logan and Nusse, 2004), which connects another component of WNT signaling into this pathway. We have previously identified the WNT signaling components $\beta$-catenin and the TCF/LEF TFs, TCF7L1 and TCF7L2, as being differentially expressed during HC regeneration (Hawkins et al., 2007). In the present study, three additional WNT signaling components, WNT4, WNT9B, and WNT16, were differentially expressed in siRNA treatments for CEBPG and LRP5. Canonical WNT signaling is transduced through the frizzled family of receptors and LRP5/LRP6 coreceptors, leading to 
activation of the $\beta$-catenin signaling cascade (Clevers, 2006). In our previous study, we determined that $\beta$-catenin is upregulated during $\mathrm{HC}$ regeneration, and in the current study, we demonstrate that siRNA knockdowns of $\beta$-catenin inhibit SE proliferation.

This study represents the first large-scale characterization of genes that are necessary for regenerative proliferation of avian SCs. It is also the first study of pathway analysis and identification in this system. As larger transcriptome datasets are generated, the types of methods described here should be applicable to identifying critical proliferative and differentiation genes and pathways in the regenerating SEs of the avian inner ear. In the longer term, these observations can be compared, contrasted, and applied to the mitotically arrested mammalian inner ear SEs with a view to replenishing damaged HC.

\section{References}

Adam J, Myat A, Le Roux I, Eddison M, Henrique D, Ish-Horowicz D, Lewis J (1998) Cell fate choices and the expression of Notch, Delta and Serrate homologues in the chick inner ear: parallels with Drosophila sense-organ development. Development 125:4645-4654.

Assi K, Pillai R, Gómez-Muñoz A, Owen D, Salh B (2006) The specific JNK inhibitor SP600125 targets tumour necrosis factor-alpha production and epithelial cell apoptosis in acute murine colitis. Immunology 118:112-121.

Bennett BL, Sasaki DT, Murray BW, O'Leary EC, Sakata ST, Xu W, Leisten JC, Motiwala A, Pierce S, Satoh Y, Bhagwat SS, Manning AM, Anderson DW (2001) SP600125, an anthrapyrazolone inhibitor of Jun N-terminal kinase. Proc Natl Acad Sci U S A 98:13681-13686.

Brivanlou AH, Darnell JE Jr (2002) Signal transduction and the control of gene expression. Science 295:813-818.

Chang J, Sonoyama W, Wang Z, Jin Q, Zhang C, Krebsbach PH, Giannobile W, Shi S, Wang CY (2007) Noncanonical Wnt-4 signaling enhances bone regeneration of mesenchymal stem cells in craniofacial defects through activation of p38 MAPK. J Biol Chem 282:30938-30948.

Chen P, Segil N (1999) p27(Kip1) links cell proliferation to morphogenesis in the developing organ of Corti. Development 126:1581-1590.

Clevers H (2006) Wnt/ $\beta$-catenin signaling in development and disease. Cell 127:469-480

Corwin JT, Cotanche DA (1988) Regeneration of sensory hair cells after acoustic trauma. Science 240:1772-1774.

Cotanche DA, Kaiser CL (2010) Hair cell fate decisions in cochlear development and regeneration. Hear Res 266:18-25.

Czerny T, Bouchard M, Kozmik Z, Busslinger M (1997) The characterization of novel Pax genes of the sea urchin and Drosophila reveal an ancient evolutionary origin of the Pax2/5/8 subfamily. Mech Dev 67:179-192.

Davydov IV, Bohmann D, Krammer PH, Li-Weber M (1995) Cloning of the cDNA encoding human C/EBP [gamma], a protein binding to the PRE-I enhancer element of the human interleukin-4 promoter. Gene 161:271-275.

Ekins S, Bugrim A, Brovold L, Kirillov E, Nikolsky Y, Rakhmatulin E, Sorokina S, Ryabov A, Serebryiskaya T, Melnikov A, Metz J, Nikolskaya T (2006) Algorithms for network analysis in systems-ADME/Tox using the MetaCore and MetaDrug platforms. Xenobiotica 36:877-901.

Elbashir SM, Harborth J, Weber K, Tuschl T (2002) Analysis of gene function in somatic mammalian cells using small interfering RNAs. Methods 26:199-213.

Frolenkov GI, Belyantseva IA, Friedman TB, Griffith AJ (2004) Genetic insights into the morphogenesis of inner ear hair cells. Nat Rev Genet 5:489-498.

Hawkins RD, Bashiardes S, Helms CA, Hu L, Saccone NL, Warchol ME, Lovett M (2003) Gene expression differences in quiescent versus regenerating hair cells of avian sensory epithelia: implications for human hearing and balance disorders. Hum Mol Genet 12:1261-1272.

Hawkins RD, Bashiardes S, Powder KE, Sajan SA, Bhonagiri V, Alvarado DM, Speck J, Warchol ME, Lovett M (2007) Large scale gene expression profiles of regenerating inner ear sensory epithelia. PLoS ONE 2:e525.

Hayward P, Kalmar T, Arias AM (2008) Wnt/Notch signalling and information processing during development. Development 135:411-424.

Heo YS, Kim SK, Seo CI, Kim YK, Sung BJ, Lee HS, Lee JI, Park SY, Kim JH,
Hwang KY, Hyun YL, Jeon YH, Ro S, Cho JM, Lee TG, Yang CH (2004) Structural basis for the selective inhibition of JNK1 by the scaffolding protein JIP1 and SP600125. EMBO J 23:2185-2195.

Hinck L, Nelson WJ, PapkoffJ (1994) Wnt-1 modulates cell-cell adhesion in mammalian cells by stabilizing beta-catenin binding to the cell adhesion protein cadherin. J Cell Biol 124:729-741.

International Chicken Genome Sequencing Consortium (2004) Sequence and comparative analysis of the chicken genome provide unique perspectives on vertebrate evolution. Nature 432:695-716.

Jørgensen JM, Mathiesen C (1988) The avian inner ear. Continuous production of hair cells in vestibular sensory organs, but not in the auditory papilla. Naturwissenschaften 75:319-320.

Kanzaki S, Beyer LA, Swiderski DL, Izumikawa M, Stöver T, Kawamoto K, Raphael Y (2006) p27Kip1 deficiency causes organ of Corti pathology and hearing loss. Hear Res 214:28-36.

Kelley MW (2006) Regulation of cell fate in the sensory epithelia of the inner ear. Nat Rev Neurosci 7:837-849.

Kozmik Z, Holland ND, Kalousova A, Paces J, Schubert M, Holland LZ (1999) Characterization of an amphioxus paired box gene, AmphiPax2/ 5/8: developmental expression patterns in optic support cells, nephridium, thyroid-like structures and pharyngeal gill slits, but not in the midbrain-hindbrain boundary region. Development 126:1295-1304.

Lanford PJ, Lan Y, Jiang R, Lindsell C, Weinmaster G, Gridley T, Kelley MW (1999) Notch signalling pathway mediates hair cell development in mammalian cochlea. Nat Genet 21:289-292.

Ledford AW, Brantley JG, Kemeny G, Foreman TL, Quaggin SE, Igarashi P, Oberhaus SM, Rodova M, Calvet JP, Vanden Heuvel GB (2002) Deregulated expression of the homeobox gene Cux-1 in transgenic mice results in downregulation of p27kip1 expression during nephrogenesis, glomerular abnormalities, and multiorgan hyperplasia. Developmental Biology 245:157-171

Ling MT, Wang X, Tsao SW, Wong YC (2002) Down-regulation of Id-1 expression is associated with TGF $\beta 1$-induced growth arrest in prostate epithelial cells. Biochim Biophys Acta 1570:145-152.

Logan CY, Nusse R (2004) The Wnt Signaling Pathway in Development and Disease. Annu Rev Cell Dev Biol 20:781-810.

Löwenheim H, Furness DN, Kil J, Zinn C, Gültig K, Fero ML, Frost D, Gummer AW, Roberts JM, Rubel EW, Hackney CM, Zenner HP (1999) Gene disruption of p27Kip1 allows cell proliferation in the postnatal and adult organ of Corti. Proc Natl Acad Sci U S A 96:4084-4088.

Lyons JP, Mueller UW, Ji H, Everett C, Fang X, Hsieh JC, Barth AM, McCrea PD (2004) Wnt- 4 activates the canonical $\beta$-catenin-mediated Wnt pathway and binds Frizzled-6 CRD: functional implications of Wnt $/ \beta$ catenin activity in kidney epithelial cells. Exp Cell Res 298:369-387.

Mansouri A, Hallonet M, Gruss P (1996) Pax genes and their roles in cell differentiation and development. Curr Opin Cell Biol 8:851-857.

Medina KL, Singh H (2005) Genetic networks that regulate B lymphopoiesis. Curr Opin Hematol 12:203-209.

Meixner A, Karreth F, Kenner L, Wagner EF (2004) JunD regulates lymphocyte proliferation and $\mathrm{T}$ helper cell cytokine expression. EMBO J 23:1325-1335.

Messina DN, Glasscock J, Gish W, Lovett M (2004) An ORFeome-based analysis of human transcription factor genes and the construction of a microarray to interrogate their expression. Genome Res 14:2041-2047.

Michl P, Ramjaun AR, Pardo OE, Warne PH, Wagner M, Poulsom R, D’Arrigo C, Ryder K, Menke A, Gress T, Downward J (2005) CUTL1 is a target of TGF $\beta$ signaling that enhances cancer cell motility and invasiveness. Cancer Cell 7:521-532.

Miyakoshi T, Takei M, Kajiya H, Egashira N, Takekoshi S, Teramoto A, Osamura RY (2008) Expression of Wnt4 in human pituitary adenomas regulates activation of the $\beta$-catenin-independent pathway. Endocr Pathol 19:261-273.

Morrison A, Hodgetts C, Gossler A, Hrabé de Angelis M, Lewis J (1999) Expression of Delta1 and Serrate1 (Jagged1) in the mouse inner ear. Mech Dev 84:169-172.

Noll M (1993) Evolution and role of Pax genes. Curr Opin Genet Dev 3:595-605.

Ramji DP, Foka P (2002) CCAAT/enhancer-binding proteins: structure, function and regulation. Biochem J 365:561-575.

Ryals BM, Rubel EW (1988) Hair cell regeneration after acoustic trauma in adult Coturnix quail. Science 240:1774-1776. 
Shaulian E, Karin M (2002) AP-1 as a regulator of cell life and death. Nat Cell Biol 4:E131-E136.

Shaulian E, Schreiber M, Piu F, Beeche M, Wagner EF, Karin M (2000) The mammalian UV response: c-Jun induction is required for exit from p53imposed growth arrest. Cell 103:897-907.

Sienknecht UJ, Fekete DM (2008) Comprehensive Wnt-related gene expression during cochlear duct development in chicken. J Comp Neurol 510:378-395.

Sienknecht UJ, Fekete DM (2009) Mapping of Wnt, frizzled, and Wnt inhibitor gene expression domains in the avian otic primordium. J Comp Neurol 517:751-764.

Singh H, Medina KL, Pongubala JM (2005) Contingent gene regulatory networks and B cell fate specification. Proc Natl Acad Sci U S A 102:4949-4953.

Stark K, Vainio S, Vassileva G, McMahon AP (1994) Epithelial transformation of metanephric mesenchyme in the developing kidney regulated by Wnt-4. Nature 372:679-683.

Stevens CB, Davies AL, Battista S, Lewis JH, Fekete DM (2003) Forced activation of Wnt signaling alters morphogenesis and sensory organ identity in the chicken inner ear. Dev Biol 261:149-164.

Stone JS, Cotanche DA (2007) Hair cell regeneration in the avian auditory epithelium. Int J Dev Biol 51:633-647.

Tam WF, Gu T-L, Chen J, Lee BH, Bullinger L, Fröhling S, Wang A, Monti S, Golub TR, Gilliland DG (2008) Id1 is a common downstream target of oncogenic tyrosine kinases in leukemic cells. Blood 112:1981-1992.

Torban E, Dziarmaga A, Iglesias D, Chu LL, Vassilieva T, Little M, Eccles M, Discenza M, Pelletier J, Goodyer P (2006) PAX2 activates WNT4 expression during mammalian kidney development. J Biol Chem 281: 12705-12712.

Wada H, Saiga H, Satoh N, Holland PW (1998) Tripartite organization of the ancestral chordate brain and the antiquity of placodes: insights from ascidian Pax-2/5/8, Hox and Otx genes. Development 125:1113-1122.

Warchol ME (1999) Immune cytokines and dexamethasone influence sensory regeneration in the avian vestibular periphery. J Neurocytol 28:889-900.

Warchol ME (2001) Lectin from Griffonia simplicifolia identifies an immature-appearing subpopulation of sensory SHC in the avian utricle. J Neurocytol 30:253-264.

Warchol ME (2002) Cell density and N-cadherin interactions regulate cell proliferation in the sensory epithelia of the inner ear. J Neurosci 22:2607-2616.

Warchol ME, Corwin JT (1996) Regenerative proliferation in organ cultures of the avian cochlea: identification of the initial progenitors and determination of the latency of the proliferative response. J Neurosci 16:5466-5477.

Weisleder P, Rubel EW (1993) Hair cell regeneration after streptomycin toxicity in the avian vestibular epithelium. J Comp Neurol 331:97-110.

White PM, Doetzlhofer A, Lee YS, Groves AK, Segil N (2006) Mammalian cochlear supporting cells can divide and trans-differentiate into hair cells. Nature 441:984-987.

Yang X, Menon S, Lykke-Andersen K, Tsuge T, Di Xiao, Wang X, RodriguezSuarez RJ, Zhang H, Wei N (2002) The COP9 signalosome inhibits p27kip1 degradation and impedes G1-S phase progression via deneddylation of SCF Cull. Curr Biol 12:667-672.

Zheng JL, Shou J, Guillemot F, Kageyama R, Gao WQ (2000) Hes1 is a negative regulator of inner ear hair cell differentiation. Development 127:4551-4560.

Zine A, Aubert A, Qiu J, Therianos S, Guillemot F, Kageyama R, de Ribaupierre F (2001) Hes1 and Hes5 activities are required for the normal development of the hair cells in the mammalian inner ear. J Neurosci 21: $4712-4720$. 